\title{
Anesthesia for endovascular treatment of acute ischemic stroke
}

Michael T. Froehler, $\mathrm{MD}, \mathrm{PhD}$

Johanna T. Fifi, MD Arshad Majid, MD

Archit Bhatt, MD Mingwen Ouyang, MD

David L. McDonagh, MD

Correspondence \& reprint requests to Dr. McDonagh: david.mcdonagh@duke.edu

\section{ABSTRACT}

The initial treatment of patients with acute ischemic stroke (AIS) focuses on rapid recanalization, which often includes the use of endovascular therapies. Endovascular treatment depends upon micronavigation of catheters and devices into the cerebral vasculature, which is easier and safer with a motionless patient. Unfortunately, many stroke patients are unable to communicate and sufficiently cooperate with the procedure. Thus, general anesthesia (GA) with endotracheal intubation provides an attractive means of keeping the patient comfortable and motionless during a procedure that could otherwise be lengthy and uncomfortable. However, several recent retrospective studies have shown an association between GA and poorer outcomes in comparison with conscious sedation for endovascular treatment of AIS, though prospective studies are lacking. The underlying reasons why GA might produce a worse outcome are unknown but may include hemodynamic instability and hypotension, delays in treatment, prolonged intubation with or without neuromuscular blockade, or even neurotoxicity of the anesthetic agent itself. Currently, the choice between GA and conscious sedation should be tailored to the individual patient, on the basis of neurologic deficits, airway and hemodynamic status, and treatment plan. The use of institutional treatment protocols may best support efficient and effective care for AIS patients undergoing endovascular therapy. Important components of such protocols would include parameters to choose anesthetic modality, timeliness of induction, blood pressure goals, minimization of neuromuscular blockade, and planned extubation at the end of the procedure. Neurology ${ }^{\circledR}$ 2012;79 (Suppl 1):S167-S173

\section{GLOSSARY}

AIS = acute ischemic stroke; $\mathbf{C l}=$ confidence interval; $\mathbf{C S}=$ conscious sedation; $\mathbf{G A}=$ general anesthesia; $\mathbf{O R}=$ odds ratio; tPA = tissue plasminogen activator

The goal of early therapy for acute ischemic stroke (AIS) is to restore perfusion to ischemic areas of the brain. The introduction of IV fibrinolysis was a tremendous step forward in emergency patient care. ${ }^{1}$ However, despite current professional education programs, enhanced public awareness, and integrated stroke care, only 3\% to $8.5 \%$ of stroke patients are treated with IV tissue plasminogen activator (tPA). ${ }^{2}$ Furthermore, fewer than half of patients with large-artery occlusions who are treated with tPA experience recanalization from thrombolysis. ${ }^{3}$ Thus, there is a need for additional reperfusion strategies.

Endovascular therapy offers a more direct approach to the occlusive lesion. Furlan et al. ${ }^{4}$ initially showed that the intra-arterial administration of thrombolytics led to improved outcomes in patients with large-artery occlusions. Later, mechanical thrombectomy was shown to be more effective in terms of recanalization, ${ }^{5,6}$ with more recent success rates of $81 \%$ to $84 \% .^{7}$ Currently, there are an array of endovascular treatment options, including intra-arterial pharmacologic fibrinolysis, guidewire maceration, clot retrieval, thrombus aspiration, angioplasty, and stenting. Choosing from the available options for endovascular stroke treatment is difficult and is usually made on a case-by-case basis, as there are often important technical differences

From the Neuro Interventional Service (M.T.F.), Department of Neurology, University of Iowa Hospitals and Clinics, Iowa City; Departments of Neurology and Radiology (J.T.F.), St. Luke's-Roosevelt Hospital, New York, NY; Hyper Acute Stroke Research Centre (A.M.), Department of Neurology, Salford Royal NHS Foundation Trust, Salford, UK; Department of Neurosciences (A.B.), Spectrum Health Medical Group, Grand Rapids, MI; Department of Anesthesiology (M.O.), Nanfang Hospital, Southern Medical University, Guangzhou, China; and Division of Neuroanesthesiology (D.L.M.), Duke University Medical Center, Durham, NC.

Go to Neurology.org for full disclosures. Disclosures deemed relevant by the authors, if any, are provided at the end of this article. 
between devices and their use. For example, some devices may require more precision in their deployment, others may cause more patient discomfort, and some may require longer procedure times.

Patients with stroke often have significant neurologic impairment; they may be aphasic and unable to communicate, may be paretic, or may experience vertigo - any of which may cause significant distress. This can make it difficult for the patient to tolerate a procedure that requires lying still for a prolonged period of time. And if the patient is unable to remain motionless, it can cause significant degradation in image quality, inability to utilize roadmap functions, and even trauma and vessel damage related to movement of the catheter. To address these considerations, anesthesia is often utilized in neurointervention. Anesthesia may come in the form of conscious sedation (CS), with administration of low-dose analgesics and hypnotics that may improve the comfort of the patient but may not be adequate to fully immobilize the patient. General anesthesia (GA) with endotracheal intubation allows for a completely motionless patient but may be associated with significant disadvantages.

In particular, several recent studies have demonstrated worse outcomes after endovascular treatment in AIS patients with GA, in comparison with CS. ${ }^{8-10}$ If this finding is true, then the choice of anesthesia during acute stroke treatment may have important ramifications. Thus, we shall first review the recent clinical data, before speculating on the potential mechanisms underlying worse outcomes with GA, which might include hemodynamic changes, neurotoxicity, delays in treatment, or prolonged intubation. A better understanding of these and other considerations should allow the development of rational protocols for the use of anesthesia in patients with AIS.

Clinical studies. There have been no prospective, randomized studies comparing GA with CS in endovascular treatment of AIS or of any other cerebrovascular disease. However, several recently published retrospective studies have provided some compelling data and ignited considerable debate on this issue. ${ }^{11,12}$
Currently, it appears that endovascular neurologists are mixed in their use of GA vs CS for patients undergoing interventional therapy for AIS. In a recent survey of the members of the Society of Vascular and Interventional Neurologists, McDonagh et al. ${ }^{13}$ found that only $6 \%$ of the respondents used GA exclusively. However, $55 \%$ of respondents believed that GA is mandatory when using mechanical thrombectomy. This preference for GA was based on the assumption that limiting movement makes the interventional procedure safer and more efficacious. Although GA and immobility allow greater image quality and decrease procedural time, the greatest perceived limitation was a delay in starting the procedure.

The relationship between periprocedural sedation and outcome has been assessed by 3 recent studies. Nichols et al. ${ }^{8}$ studied the sedation practices in the Interventional Management of Stroke II Trial. Of 81 patients, sedation data were available for 75 . A sedation classification scale was used to classify the extent of sedation used: $1=$ no sedation, $2=$ mild sedation, $3=$ heavy sedation, and $4=$ pharmacologic paralysis. Fifty-three percent $(\mathrm{n}=40)$ were given no sedation (grade 1) and 23\% ( $\mathrm{n}=17)$ were intubated/ paralyzed (grade 4). Patients in the higher sedation categories had higher baseline NIH Stroke Scale scores, suggesting more severe baseline stroke severity. Patients in lower sedation categories had better outcomes, more frequent reperfusion rates, and lower mortality. When accounting for baseline neurologic status with use of multivariate analysis, mild or no sedation (grade 1 or 2) was associated with a good clinical outcome (odds ratio [OR] 5.7; 95\% confidence interval [CI] 1.5-12.3), and heavy sedation or paralysis (grade 3 or 4) was an independent predictor of death (OR 5.0; 95\% CI 1.3-18.7).

Jumaa et al. ${ }^{9}$ retrospectively reviewed 126 patients who had received endovascular therapy for AIS due to middle cerebral artery occlusion. Level of sedation was classified as intubated $(42 \%)$ vs nonintubated (58\%). Nonintubation was associated with shorter ICU stays ( 3.2 vs 6.5 days; $p=0.0008$ ), lower infarct volume (OR $0.25 ; p=0.004)$, good clinical outcome (OR 3.06; $p=0.042$ ), and lower inhospital mortality (OR $0.32 ; p=0.011$ ). A nonsignificant difference in complications was observed, with $6 \%$ in the intubated group and $15 \%$ in the nonintubated group ( $p=0.13$ ).

The largest study was performed by Abou-Chebl et al., ${ }^{10}$ who recently reported the results of a multicenter, retrospective review of 980 endovascular acute stroke cases. GA was used in $44 \%$ of all patients, and these patients were more likely to have carotid terminus occlusions and higher NIH Stroke Scale scores. The intracranial hemorrhage rate was no differ- 
ent between GA and CS. However, GA was an independent predictor of poor neurologic outcome (OR 2.33; 95\% CI 1.63-3.44) and higher mortality (OR 1.68; 95\% CI 1.23-2.30) in multivariate analyses.

Limited published data suggest that CS may be adequate and safe in patients undergoing neurointerventional procedures for diseases other than AIS. Ogilvy et al. ${ }^{14}$ reported using CS in $92.2 \%$ of elective aneurysm embolizations in 340 patients, with low morbidity and mortality rates and short hospital stays. Additionally, complications and effectiveness were similar between GA and CS among patients undergoing angioplasty and stenting for intracranial stenosis. ${ }^{15}$ Of course, patients undergoing these elective treatments are typically much more comfortable and cooperative than patients with AIS, and these procedures are not performed in the emergent setting. Nonetheless, these reports highlight the feasibility of performing neurointerventional procedures in the nonanesthetized patient.

These retrospective data provide some evidence that outcomes in endovascular treatment of AIS may be worse with the use of GA. Of course, prospective data will be needed to definitively address this issue, although recruitment in a randomized trial may be difficult. But if the current data from these 3 trials are accurate, what might be the reason for the difference in outcomes between GA and CS? Some possibilities might include hemodynamic changes, delays in treatment, prolonged intubation, neuromuscular blockade, and neurotoxicity of the anesthetic agents themselves.

Hemodynamic effects of GA. GA has a range of systemic and cerebral effects, but probably the most unwelcome effect in acute stroke is hypotension. In the setting of AIS, an occluded artery causes focal cerebral ischemia, and a reduction in systemic blood pressure (cerebral perfusion pressure) may lead to reduction in collateral perfusion, ${ }^{16}$ which could hasten the progression to complete infarction. ${ }^{17}$ Typically, the most pronounced drop in blood pressure occurs immediately after induction. This decline in blood pressure is associated with lower baseline blood pressure, the use of certain anesthetic agents, and general health status. ${ }^{18}$ Postinduction hypotension, even in elective surgery, has been associated with prolonged hospital stays and increased mortality. ${ }^{18}$ Thus, when GA is used in acute stroke patients, blood pressure should be strictly controlled, particularly at the time of induction, with use of predefined parameters but accounting for the baseline blood pressure, stroke syndrome, and the patient's general health status.

In particular, the patient's baseline blood pressure is of critical importance, as cerebral blood flow is autoregulated only within a limited range, and hypo- tension beyond this range can lead to cerebral ischemia, especially in the setting of stroke and decreased collateral availability. Therefore, any blood pressure reduction at the time of anesthesia induction could impair potentially important collateral perfusion. The safest approach given the unknown level of risk with blood pressure reduction is to assume that the patient has tenuous collateral perfusion and to keep the blood pressure at the preinduction baseline. In practical terms, this translates into maintaining hypertension during the AIS intervention.

Although all anesthetic agents cause some hypotension, they vary in their effects on the cerebral vasculature and intracranial pressure. Specifically, the halogenated inhalational anesthetic agents (isoflurane, sevoflurane, and desflurane) are cerebral vasodilators and do not maintain the normal coupling of cerebral blood flow with cerebral metabolic rate. ${ }^{19-21}$ Thus, although they suppress the cerebral metabolic rate, they cause a relative cerebral hyperemia. This can be a significant concern in patients with elevated intracranial pressure, although not in the majority of AIS patients who have normal intracranial pressure. It should be noted that controlled ventilation with hypocapnia can offset this vasodilatory effect of the halogenated inhaled anesthetics. ${ }^{22}$

In contrast to the halogenated agents, propofol better preserves cerebral autoregulation. ${ }^{19,23}$ Therefore, as the cerebral metabolic rate is reduced with propofol, the cerebral blood volume is reduced proportionately. Nitrous oxide should be avoided in acute stroke interventions because of concerns for exacerbating any cerebrovascular air emboli entrained during the procedure. ${ }^{24,25}$

Both the halogenated anesthetic agents and propofol cause dose-dependent systemic hypotension due to vasodilatation, ${ }^{21,24}$ which is particularly pronounced at the time of induction. Propofol causes more hypotension postinduction than other induction agents such as etomidate, ${ }^{18}$ whose use may be preferred in the setting of AIS. This drop in cerebral perfusion pressure often necessitates the concomitant use of vasopressor agents. Unfortunately, we have no adequate point-of-care cerebral perfusion monitors to guide hemodynamic therapies intraoperatively during AIS interventions. Therefore, it is critical to determine blood pressure parameters before induction and to rapidly correct hypotension with pressors, on the basis of predefined blood pressure goals. Finally, other factors that could further contribute to excessive vasoconstriction or vasodilation, such as hypocapnia or hypercapnia, should be avoided.

Analgesia is an important component of GA and of CS. An opioid is typically employed for this purpose, and short-acting opioids such as remifentanil, 
because of its lack of accumulation (i.e., lack of context sensitivity), are well suited to acute stroke interventions. Hypotension is again a side effect that must be monitored closely and counteracted with pressor agents such as phenylephrine, norepinephrine, or ephedrine.

Logistical considerations. The emergent delivery of endovascular therapy to an occluded intracranial vessel requires an efficient health care delivery system involving multiple clinical services within the hospital working together. Orchestrating care among the emergency department, imaging, stroke team, and interventional service can be challenging and timeconsuming. The immediate availability of an experienced anesthesia team to provide care for patients as a Level 1 emergency is important. Unfortunately, this is not always the case in many centers. The endovascular suite is commonly located apart from main operating rooms, stretching the capability of the anesthesia team to rapidly respond, particularly at times when staffing levels are limited. The equipment and medications required for delivery of anesthetic care should be readily accessible within the neurointerventional area. Any factor that leads to a delay in the initiation of the procedure is detrimental. The familiarity of the anesthesia team with acute stroke intervention and the endovascular suite environment can vary significantly, especially during off hours. Therefore, discussion of parameters such as blood pressure goals and foreseeable time course for anesthetic induction and endotracheal intubation should take place in advance.

CS is commonly used in many centers worldwide for AIS interventions. ${ }^{12,13,26}$ However, various concerns arise with the use of CS. The optimal management of sedation to produce a cooperative, nonmoving patient varies in difficulty from patient to patient. In addition, patients have typically not fasted as they would for an elective cerebral endovascular procedure. This raises concerns for pulmonary aspiration of gastric contents in the setting of sedation and supine positioning. ${ }^{27}$ The need for emergent endotracheal intubation may result from any combination of oversedation, neurologic decline, airway compromise, or agitation. Emergent conversion to GA during the endovascular procedure may result in hypoxia or aspiration and necessitates the presence of a practitioner skilled in endotracheal intubation.

General anesthesia is a logical and seemingly attractive solution to many of these issues, since the patient will be deeply sedated with a protected airway (endotracheal intubation). However, there are potentially significant downsides. As discussed, the use of GA must not delay the delivery of reperfusion therapy. In addition, there is a loss of the neurologic ex- amination and the procedure must proceed to a radiographic endpoint rather than a clinical endpoint. As discussed above, the requirement for endotracheal intubation during GA has been associated with longer intensive care unit stays, pneumonia, and increased mortality in retrospective studies. ${ }^{8,9}$ This may be due in part to the transport of patients to the intensive care unit while intubated after the procedure, leaving the weaning to occur at a later time. Development of ventilator-associated pneumonia is known to increase with longer duration of intubation. ${ }^{28}$ Extubation immediately after the procedure to allow for neurologic examination and avoid potential complications should be the goal when possible.

Procedural paralysis. The endovascular procedure requires minimal patient movement for safe, efficient delivery of catheters and devices for thrombolysis and thrombectomy, for 2 major reasons. First, patient motion creates imaging artifact, resulting in angiographic images that are difficult to interpret. Time lost repeating imaging to obtain a clear picture of the anatomy and occlusion site can add up to significant delays. Second, patient motion during critical parts of the procedure while mechanical instrumentation is in the cerebral vasculature can lead to devastating complications. For the purposes of minimizing patient motion, GA is superior to CS or monitored anesthesia care.

Anesthesiologists utilize neuromuscular blocking agents to facilitate endotracheal intubation and provide a margin of safety during these procedures, allowing optimal visualization of the cervical and intracranial vasculature. For the acute stroke patient in whom a neurologic examination is often desired immediately after the procedure, the neuromuscular blocking agent used should be readily reversible. Intermediate-acting agents such as cisatracurium, vecuronium, and rocuronium are preferred. ${ }^{21,24}$ Depth of neuromuscular blockade should be monitored during the procedure so that reversal of the blockade can be performed at the end of the procedure and ongoing endotracheal intubation can be avoided. In addition, older agents such as atracurium, which is associated with histamine release (causing hypotension and decreased cerebral perfusion pressure), should be avoided. ${ }^{29}$

Anesthetic neuroprotection or neurotoxicity? To add further complexity to the question of GA in AIS interventions, we must consider longer-term effects of the anesthetic agents themselves. There is a large literature on the neuroprotective effects of anesthetic agents, spanning 3 decades, mostly relating to the barbiturates and isoflurane. ${ }^{30}$ In fact, there is strong evidence for the neuroprotective effect of isoflurane in rodent models of 
focal ischemia (i.e., acute stroke) ${ }^{31}$ Human trials of barbiturate neuroprotection after cardiac arrest ${ }^{32}$ were negative, but no human evidence exists for the neuroprotective or neurotoxic effects of general anesthetics in acute ischemic stroke.

More recently, concern has grown regarding the potential neurotoxic effects of anesthetics, particularly isoflurane. ${ }^{33-36}$ There is evidence from cell culture and rodent models that isoflurane promotes oligomerization of $\beta$-amyloid, one of the pathophysiologic processes in Alzheimer disease, and causes neurotoxicity in both neonatal and elderly animals. ${ }^{33,34}$ Nitrous oxide and ketamine have also been implicated, and data from animal studies indicate that toxicity may be mediated by NMDA receptor antagonist (e.g., ketamine)-induced vacuolization of neurons of adult and aged rodents. ${ }^{35,36}$ Human studies are under way in at-risk populations.

To our knowledge, there are no data to date regarding anesthetic neurotoxicity in acute ischemic stroke. Whether common perioperative neurologic complications such as delirium and postoperative cognitive dysfunction are related to anesthetic neurotoxicity or other factors is similarly unknown. ${ }^{37}$

It will be difficult to directly study the neuroprotective or neurotoxic effects of general anesthetics in patients with AIS. However, outcome studies in ischemic stroke populations are needed to define the shortterm and long-term impact of general anesthetics on the nervous system. In addition, animal stroke models should also be utilized to explore the potential mechanisms of neurotoxicity of anesthetic agents.

As an aside, the discussion of neuroprotection would be incomplete without considering therapeutic hypothermia. The use of GA for AIS interventions would facilitate the rapid induction of therapeutic hypothermia. However, to date, only early stage feasibility trials have been conducted, ${ }^{38}$ and there is currently no convincing human evidence to suggest that AIS patients benefit from cooling. Current goals are to maintain normothermia, while avoiding hyperthermia, in AIS interventions.

DISCUSSION Acute stroke therapy has evolved significantly. Entire systems of care have developed to support the delivery of recanalization therapies, which have included physician, hospital, corporate, and legislative efforts. ${ }^{39,40}$ Although these systems have certainly improved the structure of care and resources available to those of us who treat ischemic stroke, the treatment of each individual patient still brings new challenges to the rapid delivery of appropriate therapy. One such challenge is the decision regarding anesthesia for AIS patients undergoing endovascular treatment. Making this decision involves an assessment of neurologic status, airway, ability to cooperate with the procedure, anticipated technique and procedure time, planned postprocedure care, and other factors. And beyond these individual patient factors, we must consider the general risks of GA, including delay to treatment, hemodynamic instability, and the loss of the neurologic examination during the procedure. In light of recent data, we must also consider the possibility that the use of GA may be associated with poorer outcomes. Randomized controlled trials of the use of GA in AIS interventions would provide the highest level of evidence to guide therapy. However, in the interim, the inclusion of sedation type, factors influencing choice of sedation, and complications related to sedative modality should be prospectively gathered in any AIS intervention trials. This will provide intermediatelevel evidence that is superior to what is currently available in regard to the impact of GA on outcome after AIS interventions.

Until we have better evidence to guide us, we must individualize choice of anesthesia to each patient. GA may be more appropriate for patients with severe deficits, airway compromise, or bulbar dysfunction. CS may be more appropriate for patients with milder deficits or those with tenuous hemodynamic status. To minimize the potentially negative effects of GA with endotracheal intubation, the neurointerventionalist and anesthesiologist should plan to extubate at the end of the case unless there is a contraindication, and agree upon hemodynamic parameters before the start of the case. Ideally, these goals would be supported by institutional treatment protocols that would avoid the need for lengthy planning and discussion prior to the start of each case. Indeed, the use of GA vs CS can be based on a protocol that incorporates clinical status, anticipated procedural technique, and planned postprocedure care. The use of standardized protocols in treating for ischemic stroke patients does improve their care, ${ }^{39}$ and we would advocate the use of an anesthesia protocol to further support safe and efficient endovascular treatment for patients with AIS.

\section{AUTHOR CONTRIBUTIONS}

Dr. Froehler and Dr. Fifi: drafting/revising the manuscript, study concept or design, analysis or interpretation of data. Dr. Majid: drafting/revising the manuscript. Dr. Bhatt and Dr. Ouyang: drafting/revising the manuscript, study supervision. Dr. McDonagh: drafting/revising the manuscript, study concept or design, analysis or interpretation of data, acquisition of data.

\section{ACKNOWLEDGMENT}

The authors thank Dr. Harold P. Adams for comments on the manuscript.

\section{DISCLOSURE}

Dr. Froehler has received funding from the A.P. Giannini Foundation and from the US Food and Drug Administration as a medical officer. Dr. 
Fifi received a speaker's honorarium and travel expenses from Penumbra, Inc. Dr. Majid has served as an Associate Editor for Neurohospitalist and served on the speakers bureau for Boehringer Ingelheim. Dr. Bhatt and Dr. Ouyang report no disclosures. Dr. McDonagh has served on the Editorial Board of Journal of Neurosurgical Anesthesiology; received consulting fees from Cephalogics Corporation, LLC; and received research support from the Alzheimer's Disease Research Foundation. Go to Neurology. org for full disclosures.

Received September 21, 2011. Accepted in final form December 1, 2011.

\section{REFERENCES}

1. The National Institute of Neurological Disorders and Stroke rt-PA Stroke Study Group. Tissue plasminogen activator for acute ischemic stroke. N Engl J Med 1995;333: 1581-1587.

2. Reeves MJ, Arora S, Broderick JP, et al. Acute stroke care in the US: results from 4 pilot prototypes of the Paul Coverdell National Acute Stroke Registry. Stroke 2005;36: 1232-1240.

3. Rha JH, Saver JL. The impact of recanalization on ischemic stroke outcome: a meta-analysis. Stroke 2007;38: 967-973.

4. Furlan A, Higashida R, Wechsler L, et al. Intra-arterial prourokinase for acute ischemic stroke: the PROACT II study: a randomized controlled trial: Prolyse in Acute Cerebral Thromboembolism. JAMA 1999;282:2003-2011.

5. Smith WS, Sung G, Starkman S, et al. Safety and efficacy of mechanical embolectomy in acute ischemic stroke: results of the MERCI trial. Stroke 2005;36:1432-1438.

6. The penumbra pivotal stroke trial: safety and effectiveness of a new generation of mechanical devices for clot removal in intracranial large vessel occlusive disease. Stroke 2009; 40:2761-2768.

7. Costalat V, Machi P, Lobotesis K, et al. Rescue, combined, and stand-alone thrombectomy in the management of large vessel occlusion stroke using the solitaire device: a prospective 50-patient single-center study: timing, safety, and efficacy. Stroke 2011;42:1929-1935.

8. Nichols C, Carrozzella J, Yeatts S, Tomsick T, Broderick J, Khatri P. Is peri-procedural sedation during acute stroke therapy associated with poorer functional outcomes? J Neurointerv Surg 2010;2:67-70.

9. Jumaa MA, Zhang F, Ruiz-Ares G, et al. Comparison of safety and clinical and radiographic outcomes in endovascular acute stroke therapy for proximal middle cerebral artery occlusion with intubation and general anesthesia versus the nonintubated state. Stroke 2010;41:1180-1184.

10. Abou-Chebl A, Lin R, Hussain MS, et al. Conscious sedation versus general anesthesia during endovascular therapy for acute anterior circulation stroke: preliminary results from a retrospective, multicenter study. Stroke 2010;41: $1175-1179$

11. Molina CA, Selim MH. General or local anesthesia during endovascular procedures: sailing quiet in the darkness or fast under a daylight storm. Stroke 2010;41:2720-2721.

12. Gupta R. Local is better than general anesthesia during endovascular acute stroke interventions. Stroke 2010;41: 2718-2719.

13. McDonagh DL, Olson DM, Kalia JS, Gupta R, AbouChebl A, Zaidat OO. Anesthesia and sedation practices among neurointerventionalists during acute ischemic stroke endovascular therapy. Front Neurol 2010;1:118.

14. Ogilvy CS, Yang X, Jamil OA, et al. Neurointerventional procedures for unruptured intracranial aneurysms under procedural sedation and local anesthesia: a large-volume, single-center experience. J Neurosurg 2011;114:120-128.

15. Chamczuk AJ, Ogilvy CS, Snyder KV, et al. Elective stenting for intracranial stenosis under conscious sedation. Neurosurgery 2010;67:1189-1193.

16. Bang OY, Saver JL, Alger JR, Starkman S, Ovbiagele B, Liebeskind DS. Determinants of the distribution and severity of hypoperfusion in patients with ischemic stroke. Neurology 2008;71:1804-1811.

17. Ginsberg MD, Pulsinelli WA. The ischemic penumbra, injury thresholds, and the therapeutic window for acute stroke. Ann Neurol 1994;36:553-554.

18. Reich DL, Hossain S, Krol M, et al. Predictors of hypotension after induction of general anesthesia. Anesth Analg 2005;101:622-628.

19. Kaisti KK, Långsjö JW, Aalto $S$, et al. Effects of sevoflurane, propofol, and adjunct nitrous oxide on regional cerebral blood flow, oxygen consumption, and blood volume in humans. Anesthesiology 2003;99:603-613.

20. Dinsmore J. Anaesthesia for elective neurosurgery. $\mathrm{Br} \mathrm{J}$ Anaesth 2007;99:68-74.

21. Lee CZ, Litt L, Hashimoto T, Young WL. Physiologic monitoring and anesthesia considerations in acute ischemic stroke. J Vasc Interv Radiol 2004;15:S13-S19.

22. McCulloch TJ, Boesel TW, Lam AM. The effect of hypocapnia on the autoregulation of cerebral blood flow during administration of isoflurane. Anesth Analg 2005;100: 1463-1467.

23. Adembri C, Venturi L, Pellegrini-Giampietro DE. Neuroprotective effects of propofol in acute cerebral injury. CNS Drug Rev 2007;13:333-351.

24. Young WL. Anesthesia for endovascular neurosurgery and interventional neuroradiology. Anesthesiol Clin 2007;25: 391-412.

25. Varma MK, Price K, Jayakrishnan V, Manickam B, Kessell G. Anaesthetic considerations for interventional neuroradiology. Br J Anaesth 2007;99:75-85.

26. Brekenfeld C, Mattle HP, Schroth G. General is better than local anesthesia during endovascular procedures. Stroke 2010;41:2716-2717.

27. Practice guidelines for preoperative fasting and the use of pharmacologic agents to reduce the risk of pulmonary aspiration: application to healthy patients undergoing elective procedures: a report by the American Society of Anesthesiologist Task Force on Preoperative Fasting. Anesthesiology 1999;90:896-905.

28. Byers JF, Sole ML. Analysis of factors related to the development of ventilator-associated pneumonia: use of existing databases. Am J Crit Care 2000;9:344-349.

29. Schramm WM, Papousek A, Michalek-Sauberer A, Czech $T$, Illievich $U$. The cerebral and cardiovascular effects of cisatracurium and atracurium in neurosurgical patients. Anesth Analg 1998;86:123-127.

30. Bickler PE, Patel PM. Anesthetic neuroprotection: some things do last. Anesthesiology 2007;106:8-10.

31. Sakai H, Sheng H, Yates RB, Ishida K, Pearlstein RD, Warner DS. Isoflurane provides long-term protection against focal cerebral ischemia in the rat. Anesthesiology 2007;106:92-99.

32. Brain Resuscitation Clinical Trial I Study Group. Randomized clinical study of thiopental loading in comatose survivors of cardiac arrest. N Engl J Med 1986;314:397-403.

33. Xie Z, Culley DJ, Dong Y, et al. The common inhalation anesthetic isoflurane induces caspase activation and in- 
creases amyloid beta-protein level in vivo. Ann Neurol 2008;64:618-627.

34. Stratmann G, Sall JW, May LD, et al. Isoflurane differentially affects neurogenesis and long-term neurocognitive function in 60-day-old and 7-day-old rats. Anesthesiology 2009;110:834-848.

35. Culley DJ, Raghavan SV, Waly M, et al. Nitrous oxide decreases cortical methionine synthase transiently but produces lasting memory impairment in aged rats. Anesth Analg 2007;105:83-88.

36. Zou X, Patterson TA, Sadovova N, et al. Potential neurotoxicity of ketamine in the developing rat brain. Toxicol Sci 2009;108:149-158.
37. Monk TG, Price CC. Postoperative cognitive disorders. Curr Opin Crit Care 2011;17:376-381.

38. Yenari MA, Hemmen TM. Therapeutic hypothermia for brain ischemia: where have we come and where do we go? Stroke 2010;41:S72-S74.

39. Hachinski V, Donnan GA, Gorelick PB, et al. Stroke: working toward a prioritized world agenda. Stroke 2010; 41:1084-1099.

40. Fonarow GC, Smith EE, Saver JL, et al. Improving doorto-needle times in acute ischemic stroke: the design and rationale for the American Heart Association/American Stroke Association's Target: Stroke Initiative. Stroke 2011; 42:2983-2989. 


\section{Neurology}

\section{Anesthesia for endovascular treatment of acute ischemic stroke \\ Michael T. Froehler, Johanna T. Fifi, Arshad Majid, et al. Neurology 2012;79;S167-S173 \\ DOI 10.1212/WNL.0b013e31826959c2}

This information is current as of September 24, 2012

\section{Updated Information \& Services}

References

Subspecialty Collections

Permissions \& Licensing

Reprints including high resolution figures, can be found at: http://n.neurology.org/content/79/13_Supplement_1/S167.full

This article cites 40 articles, 16 of which you can access for free at: http://n.neurology.org/content/79/13_Supplement_1/S167.full\#ref-list1

This article, along with others on similar topics, appears in the following collection(s):

\section{All Cerebrovascular disease/Stroke}

http://n.neurology.org/cgi/collection/all_cerebrovascular_disease_strok e

\section{Critical care}

http://n.neurology.org/cgi/collection/critical_care

Other cerebrovascular disease/ Stroke

http://n.neurology.org/cgi/collection/other_cerebrovascular_disease_st roke

Information about reproducing this article in parts (figures,tables) or in its entirety can be found online at:

http://www.neurology.org/about/about_the_journal\#permissions

Information about ordering reprints can be found online:

http://n.neurology.org/subscribers/advertise

Neurology ${ }^{\circledR}$ is the official journal of the American Academy of Neurology. Published continuously since 1951, it is now a weekly with 48 issues per year. Copyright Copyright (? 2012 by AAN Enterprises, Inc.. All rights reserved. Print ISSN: 0028-3878. Online ISSN: 1526-632X.

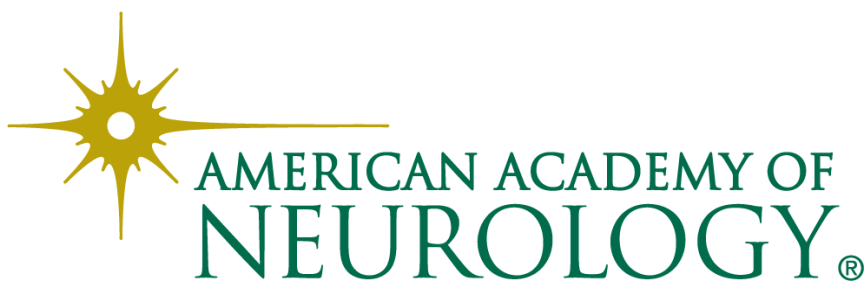

